\title{
An efficient operator splitting method for local region Chan-Vese model
}

\author{
Hui Wang ${ }^{1,2}$, Ting-Zhu Huang ${ }^{1 *}$ and Jun Liu ${ }^{1}$
}

\begin{abstract}
In this paper, we propose an efficient operator splitting method for local region Chan-Vese (C-V) model for image segmentation. Different from the C-V model, we employ the window function and absorb the local characteristics of the image for improving the C-V model, which we called the local C-V model. The local C-V model can deal with the problem of intensity inhomogeneity which widely exists in the real-world images. By employing a Laplacian operator, we present an operator splitting method to update the level set function. Firstly, we solve the proposed model for evolving the level set function, which drives the active contour to move toward the object boundaries. Secondly, we introduce the Laplacian operator to act on the level set function as a diffusion term, which could efficiently ensure the smoothness and stability and eliminate the complex process of re-initialization. Besides, we increase a new constraint term which avoids updating the level set function seriously. Furthermore, we present an extension for vector-valued images. Experiment results show that our method is competitive with application to synthetic and real-world images.
\end{abstract}

\section{Introduction}

In the field of image processing and computer vision, image segmentation is an everlasting fundamental problem. In the past decades, a large number of different approaches to segmentation have been put forward continuously $[1,2]$. the active contour model that was firstly presented by Kass et al. [3] is one of the most famous and successful models for extracting objects in image segmentation. The main idea of this model is evolving a parametric curve to extract the objects during a process of minimizing energy functional. However, this model has some intrinsic disadvantages, such as it cannot efficiently handle topological changes like splitting and merging of the evolving curve. In order to overcome this problem, the level set method [4] proposed by Osher and Sethian could easily represent the curve or surface as the zero level set of a high-dimensional function which can effectively handle topological changes. With the evolution of the level set function, the curve is moving implicitly, which promotes the combination with the active contour model. Up to now, in order to provide an effective way, active contour models $[3,5-7]$ based on the theory of curve and

\footnotetext{
*Correspondence: tingzhuhuang@126.com

1 School of Mathematical Sciences, University of Electronic Science and

Technology of China, Chengdu, Sichuan 611731, China

Full list of author information is available at the end of the article
}

surface evolutions and geometric flows have been extensively studied and successfully used in the field of image segmentation.

Generally, active contour models can be roughly categorized into two different classes: edge-based models [6,8-11] and region-based models [7,12-17]. Edge-based models use local image gradient information to attract the active contour toward the object boundaries and stop there. Geodesic active contour (GAC) model [6] is a famous example of this kind, which mainly depends on the local gradient information to control the shrinking or expanding of the contour. This kind of models is sensitive to the initial conditions and sometimes with boundary leakage problems, especially to the weak or fuzzy boundaries. Comparing with the edge-based models, region-based models aim to identify each region by introducing region descriptors to drive the contour evolution. Depending on the statistical region information, they offer advantages such as that they do not rely on any edge or gradient information and are generally robust to noise and less sensitive to the contour initialization. In this paper, we mainly focus on the region-based models.

Among the region-based models, the Mumford-Shah model [18] is well known in minimizing an energy functional to approximate the image. In the Mumford-Shah model, the image is decomposed into some regions. In this

\section{是 Springer}

(c) 2013 Wang et al: licensee Springer. This is an Open Access article distributed under the terms of the Creative Commons Attribution License (http://creativecommons.org/licenses/by/2.0), which permits unrestricted use, distribution, and reproduction in any medium, provided the original work is properly cited. 
way, each region is approximated as a smooth function. The C-V model [7], as a simplified case of the MumfordShah model, in a piecewise constant way, has achieved a good performance in two-phase image segmentation with a fast convergence rate. On the basis of the $\mathrm{C}-\mathrm{V}$ model, in $[19,20]$, the authors further generalized and proposed some variants which are called piecewise constant models. On the other hand, the energy functional of the $\mathrm{C}-\mathrm{V}$ model is non-convex, so it is prone to getting struck in undesirable local minima. In [21-23], the authors presented some convex relaxation methods. However, the $\mathrm{C}-\mathrm{V}$ model is based on the assumption that the image is statistically intensity homogeneous in each region, thus it has some limitations in actual applications. In fact, the image with intensity inhomogeneity exists widely in the real world, and it is considered as a challenging problem in image segmentation. In addition, the typical C-V model can only deal with the problem of twophase segmentation. As an extension, a multiphase level set framework [24] is presented for the multi-region image segmentation, which can be used to deal with the problem of intensity inhomogeneity. However, re-initialization is required periodically for the level set function so the computational cost is expensive. On the other hand, for the benefit of vector-valued image segmentation, in [25], the authors extended the $\mathrm{C}-\mathrm{V}$ model to the vector-valued images. In [16,17], local region information is incorporated into the active contour models; and it is worth mentioning that the local binary fitting (LBF) model, also called region-scalable fitting model, shows a better performance than the $\mathrm{C}-\mathrm{V}$ model on extracting objects to the images with intensity inhomogeneity. However, the LBF model has a large dependency on the contour initialization; especially if the initial position of the contour is far away from the objects, the LBF model may be prone to getting stuck in local minima. Apart from the LBF model, in [26-28], active contour models mainly based on the local region information are further developed and effectively used to segment the images with intensity inhomogeneity. In [29], Tao et al. integrated the multiple piecewise constant with the GAC model, which can also overcome the problem of intensity inhomogeneity and multiple objects for image segmentation. Besides, in [30], the authors integrated the local region information with the $\mathrm{C}-\mathrm{V}$ model, which is effective for the images with intensity inhomogeneity.

In the traditional level set methods, in order to keep the regularity and numerical stability during the evolving process, periodical re-initialization [31-33] as a numeric remedy is introduced to maintain the level set function regularity. However, this method is time-consuming and sometimes it may move the location of the zero level set [34]. Considering these problems, in [35-37], the authors proposed a series of variational level set methods, which can approximately maintain the signed distance property with the level set evolution. Therefore, these methods completely avoid the re-initialization procedure. Besides, in $[27,38]$, the authors used the Gaussian filtering processing to regularize the level set function.

In particular, Zhang et al. [39] proposed a reaction diffusion method, in which the level set evolving process can be divided two steps, where the re-initialization procedure is also completely unnecessary.

In this paper, we propose an efficient operator splitting method for local region $\mathrm{C}-\mathrm{V}$ model, which employs the local image region information to drive the active contour evolving. Unlike the $\mathrm{C}-\mathrm{V}$ model, we bring in a window function to calculate the local means of image intensities inside and outside the contour, respectively, and apply them to improve the $\mathrm{C}-\mathrm{V}$ model. For the sake of simplicity, we call it as local C-V model. In the level set evolving process, the local $\mathrm{C}-\mathrm{V}$ model mainly relies on the local image region information so that it is desirable to segment the images with intensity inhomogeneity. Furthermore, considering the regularity of the level set function, we present an operator splitting method to update the level set function, which performs well in maintaining its smoothness and stability. Specifically, in the first step, the level set formulation is iterated. In the second step, motivated by the relative contributions in [27,39], we introduce the Laplacian operator to act on the level set function, which forms a diffusion term to regularize the level set function. This diffusion term can ensure the smoothness and stability of the level set function, thus the costly reinitialization procedure is not essential. In addition, we increase a new constraint term, which avoids updating the level set function seriously and maintains its stability as well. Moreover, we extend our method to the vectorvalued image segmentation, as a special case, which can be used to extract the objects on the color images.

The outline of this paper is organized as follows. In Section 2, we mainly review the well-known MumfordShah model and the C-V model and its extension form on the vector-valued images. In Section 3, we first propose the local region $\mathrm{C}-\mathrm{V}$ model, and then we present an operator splitting method to realize the level set evolution and keep its smoothness and stability at the same time. Furthermore, we also extend our method to the vector-valued images. In Section 4, we carry out some experiments to demonstrate the effectiveness and performance of our method. Finally, we summarize this paper in Section 5.

\section{Previous related works}

\subsection{Mumford-Shah model and Chan-Vese model}

In [18], Mumford and Shah proposed the Mumford-Shah model. Its main idea is as follows: given an image $I$, by minimizing an energy functional to find a pair of $(u, C)$ for partitioning the image into some disjoint sub-regions, $u$ is 
an almost piecewise smooth approximation of the original image $I$, and $C$ denotes the smooth and closed contour. The energy functional is defined as

$$
E^{\mathrm{MS}}(u, C)=\int_{\Omega}|u-I|^{2} d x d y+\mu \int_{\Omega \backslash C}|\nabla u|^{2} d x d y+v|C|,
$$

where $\Omega$ defines the image domain, and $\mu$ and $\nu$ are fixed parameters, and $|C|$ is the length of the contour $C \subset \Omega$. In this energy functional, the first term is the data fidelity term. The second and third terms are the smooth term and the length restraint term of the contour $C$. In fact, because of non-convex property, it is a difficult problem to find the optimal solution of $(1)$. In $[7,24,40]$, the authors turned to simplify or modify this problem for practical applications.

Based on the Mumford-Shah model, Chan and Vese [7] considered a special case by restricting $u$ to a piecewise constant function and proposed an energy functional as follows:

$$
\begin{aligned}
E^{C V}\left(C, c_{1}, c_{2}\right)= & \mu|C|+\lambda_{1} \int_{\operatorname{inside}(C)}\left|I(x, y)-c_{1}\right|^{2} d x d y \\
& +\lambda_{2} \int_{\operatorname{Outside}(C)}\left|I(x, y)-c_{2}\right|^{2} d x d y,
\end{aligned}
$$

where $\mu \geq 0, v \geq 0, \lambda_{1}>0$, and $\lambda_{2}>0$ are parameters.

In the $\mathrm{C}-\mathrm{V}$ model (2), the combination of the last two terms is called fitting energy, which is based on the statistic region information inside and outside the contour $C$, respectively. In totality, this fitting energy plays a major role in the process of the contour evolution. In calculus of variations and level set methods, the contour $C$ is readily represented by the zero level set $\phi(x, y)=0$. Correspondingly, $\phi(x, y)>0$, if $(x, y)$ is inside $C$, and reversely $\phi(x, y)<0$, if $(x, y)$ is outside $C$. Minimizing the energy functional (2) is equivalent to solving the corresponding level set formulation as follows:

$$
\begin{aligned}
\frac{\partial \phi}{\partial t}= & \delta(\phi)\left[\mu \operatorname{div}\left(\frac{\nabla \phi}{|\nabla \phi|}\right)-\lambda_{1}(I(x, y)\right. \\
& \left.\left.-c_{1}\right)^{2}+\lambda_{2}\left(I(x, y)-c_{2}\right)^{2}\right],
\end{aligned}
$$

where $\delta$ is the Dirac function. Similarly, after fixing $\phi, c_{1}$ and $c_{2}$ are easily obtained as

$$
\begin{aligned}
& c_{1}(\phi)=\frac{\int_{\Omega} I(x, y) H(\phi(x, y)) d x d y}{\int_{\Omega} H(\phi(x, y)) d x d y}, \\
& c_{2}(\phi)=\frac{\int_{\Omega} I(x, y)(1-H(\phi(x, y))) d x d y}{\int_{\Omega}(1-H(\phi(x, y))) d x d y},
\end{aligned}
$$

where $H$ is the Heaviside function.
The $\mathrm{C}-\mathrm{V}$ model is considered as one of the most widely used models for two-phase image segmentation. One of the prominent advantages of the $\mathrm{C}-\mathrm{V}$ model is that it performs well on the images with fuzzy or even without edges. However, as a limitation, the $\mathrm{C}-\mathrm{V}$ model always supposes the image with intensity homogeneity. In fact, from Equations 4 and 5 , we can observe that $c_{1}$ and $c_{2}$ are mainly related to the global property for they rely on the region information inside and outside the contour, respectively. Without taking the local image region information into account, the $\mathrm{C}-\mathrm{V}$ model cannot effectively deal with the problem of intensity inhomogeneity. In addition, it is unable to segment the multi-region images with different intensities.

\subsection{Vector-valued Chan-Vese model}

In [25], Chan et al. presented a natural extension of the traditional $\mathrm{C}-\mathrm{V}$ model to the vector-valued images. In this extension, let $I_{i}$ be the $i$ th channel of an image on $\Omega$, with $i=1, \cdots, N$ channels, and $C$ the evolving curve. All the channels originate from the same image with some differences. Let

$$
\overline{c^{+}}=\left(c_{1}^{+}, \cdots, c_{N}^{+}\right) \text {and } \overline{c^{-}}=\left(c_{1}^{-}, \cdots, c_{N}^{-}\right)
$$

be two unknown constant vectors. Then the extension of the $\mathrm{C}-\mathrm{V}$ model to the vector case is as follows:

$$
\begin{aligned}
E\left(\overline{c^{+}}, \overline{c^{-}}, \phi\right)= & \mu|C|+\int_{\operatorname{inside}(C)} \frac{1}{N} \sum_{i=1}^{N} \lambda_{i}^{+}\left|I_{i}(x, y)-c_{1}^{+}\right|^{2} d x d y \\
& +\int_{\operatorname{Outside}(C)} \frac{1}{N} \sum_{i=1}^{N} \lambda_{i}^{-}\left|I_{i}(x, y)-c_{1}^{-}\right|^{2} d x d y
\end{aligned}
$$

where $\lambda_{i}^{+}>0$ and $\lambda_{i}^{-}>0$ are parameters for the $i$ th channel. For convenience, we call the energy functional (6) as vector-valued $\mathrm{C}-\mathrm{V}$ model. As an extension, the vectorvalued $\mathrm{C}-\mathrm{V}$ model balances the length of the contour $C$ in the image, with fitting of $I$, averaged over all channels. In this form, the model (6) can detect edges presented in the last one of the channels and not necessarily in all channels. In the level set methods, the energy functional (6) can be easily rewritten as

$$
\begin{aligned}
E\left(\overline{c^{+}, \overline{c^{-}}}, \phi\right) & =\mu \int_{\Omega} \delta(\phi(x, y))|\nabla \phi(x, y)| d x d y \\
+ & \int_{\Omega} \frac{1}{N} \sum_{i=1}^{N} \lambda_{i}^{+}\left|I_{i}(x, y)-c_{1}^{+}\right|^{2} H(\phi(x, y)) d x d y \\
+ & \int_{\Omega} \frac{1}{N} \sum_{i=1}^{N} \lambda_{i}^{-}\left|I_{i}(x, y)-c_{1}^{-}\right|^{2}(1-H(\phi(x, y))) d x d y .
\end{aligned}
$$


Similar to the C-V model in minimizing the energy functional (7) with respect to the constants $c_{i}^{+}$and $c_{i}^{-}$for $i=1, \cdots, N$, we obtain

$$
\begin{aligned}
c_{i}^{+}(\phi) & =\frac{\int_{\Omega} I_{i}(x, y) H(\phi(x, y)) d x d y}{\int_{\Omega} H(\phi(x, y)) d x d y}, \\
c_{i}^{-}(\phi) & =\frac{\int_{\Omega} I_{i}(x, y)(1-H(\phi(x, y))) d x d y}{\int_{\Omega}(1-H(\phi(x, y))) d x d y} .
\end{aligned}
$$

Then we assume that $\overline{c^{+}}$and $\overline{c^{-}}$are constant vectors, and then we minimize the energy functional (7) with respect to $\phi$ to have

$$
\begin{gathered}
\frac{\partial \phi}{\partial t}=\delta(\phi)\left[\mu \operatorname{div}\left(\frac{\nabla \phi}{|\nabla \phi|}\right)-\frac{1}{N} \sum_{i=1}^{N} \lambda_{i}^{+}\left(I_{i}(x, y)\right.\right. \\
\left.\left.-c_{i}^{+}\right)^{2}+\frac{1}{N} \sum_{i=1}^{N} \lambda_{i}^{-}\left(I_{i}(x, y)-c_{i}^{-}\right)^{2}\right] .
\end{gathered}
$$

The vector-valued $\mathrm{C}-\mathrm{V}$ model can be used to extract complete information of the image, including the missing information in one or more channels. The reason is that each single channel is insufficient for determining the final location of the evolving contour. Thus, the vector-valued $\mathrm{C}-\mathrm{V}$ model is better with the combinatorial form of all the channels. As a special example in [25], the vector-valued $\mathrm{C}-\mathrm{V}$ model is effective to segment the color images. In fact, the vector-value $\mathrm{C}-\mathrm{V}$ model inherits all the benefits of the traditional $\mathrm{C}-\mathrm{V}$ model, such as robustness to the noise and automatic detection of interior contours. Nevertheless, the vector-value $\mathrm{C}-\mathrm{V}$ model still cannot deal well with the image with intensity inhomogeneity.

\section{An efficient operator splitting method for local region Chan-Vese model}

3.1 A local region Chan-Vese model

As discussed in subsection 2.1, in the level set formulation

(3) of the $\mathrm{C}-\mathrm{V}$ model, $c_{1}$ and $c_{2}$ represent the global mean intensities of the image inside and outside the contour, respectively. As a result of this simplified representation, the $\mathrm{C}-\mathrm{V}$ model fails in segmenting the image with intensity inhomogeneity. Generally, the intensity inhomogeneity is slowly varying in the image domain. A remarkable characteristic of the image with intensity inhomogeneity is that for each point $(x, y) \in \Omega$, its intensity is not completely similar to its neighboring points, sometimes with a great deal of difference. Therefore, it is unreasonable to only use two constants $c_{1}$ and $c_{2}$ for approximating the image intensities inside and outside the contour, respectively. To address these issues, we assume that for each point $(x, y)$, in a small neighboring region, the image intensities submit to a certain probability distribution function, such as the Gaussian distribution. For the benefit of incorporating the local region information, we introduce a truncated rectangular window function $W_{k}(x, y)$, where $k$ is related to the size of this rectangular window. Then we define $c_{1}^{\mathrm{L}}$ and $c_{2}^{\mathrm{L}}$ as follows:

$$
\begin{aligned}
& c_{1}^{\mathrm{L}}=\operatorname{mean}\left(I \in\left(\{(x, y) \in \Omega \mid \phi(x, y)>0\} \cap W_{k}(x, y)\right)\right), \\
& c_{2}^{\mathrm{L}}=\operatorname{mean}\left(I \in\left(\{(x, y) \in \Omega \mid \phi(x, y)<0\} \cap W_{k}(x, y)\right)\right),
\end{aligned}
$$

where we choose the truncated rectangular Gaussian window with a standard deviation $\sigma$. Comparing with Equations 4 and 5 , it is more complex for calculating $c_{1}^{\mathrm{L}}$ and $c_{2}^{\mathrm{L}}$, which greatly relies on the size of the window function. Due to the effect of the window function, the contributions of the intensities of the neighboring points to the center point are not the same for the different distances. To deal well with the problem of the image with intensity inhomogeneity, we use $c_{1}^{\mathrm{L}}$ and $c_{2}^{\mathrm{L}}$ to replace $c_{1}$ and $c_{2}$. Therefore, the level set formulation (3) can be rewritten as

$$
\begin{gathered}
\frac{\partial \phi}{\partial t}=\delta(\phi)\left[\mu \operatorname{div}\left(\frac{\nabla \phi}{|\nabla \phi|}\right)-\lambda_{1}(I(x, y)\right. \\
\left.\left.-c_{1}^{\mathrm{L}}\right)^{2}+\lambda_{2}\left(I(x, y)-c_{2}^{\mathrm{L}}\right)^{2}\right],
\end{gathered}
$$

where $c_{1}^{\mathrm{L}}$ and $c_{2}^{\mathrm{L}}$ are with local region property of the image, thus we call Equation 13 as the local region ChanVese model. Comparing with the level set formulation (3), one of the primary advantages here is that instead of the global region information, we bring in the local image region information to drive the contour evolution. In this way, by adjusting the size and variance of the Gaussian window function, the image region with intensity inhomogeneity can be distinctively treated with the contour evolution, which greatly enhances the improvement of segmentation quality.

To solve the level set formulation (13), similarly in $[7,30]$, we use a finite difference method in numerical scheme. Then Equation 13 can be discretized as

$$
\frac{\phi_{i, j}^{n+1}-\phi_{i, j}^{n}}{\Delta t}=L\left(\phi_{i, j}^{n}\right) .
$$

In numerical implementation, we use the Neumann boundary condition. In fact, we can directly update the level set function by Equation 14 after initialization. Nevertheless, the regularity and stability of the level set function cannot be availably maintained during the evolving process.

\subsection{An operator splitting method}

In the level set methods, how to maintain the smoothness and stability is a key problem. As discussed in Section 1, the re-initialization [32] has been extensively used as a numerical remedy for maintaining the stability and the signed distance property during the level set 
evolution. However, the procedure is time-consuming, and, more importantly, it may lead to the movement of the zero level set location. In [35-37], variational level set methods are presented, all of which effectively eliminate the re-initialization procedure and improve the computational efficiency. But these methods are not easily extended to other level set methods based on partial differential equations [39], and sometimes with the boundary leakage problems, which extremely restrict their extension and utilization on the image segmentation. More specifically, it is essential and extremely important to regularize the level set function during its evolution process. In other words, for our proposed level set formulation (13), the regularization procedure of the level set function is a requisite with its evolution process. Consequently, motivated by the discussion in the works of $[27,39]$, we present an operator splitting method to evolve the level set function as follows:

- Step 1. Based on Equation 14, update the level set function by $\phi^{n+1 / 2}=\phi^{n}+\Delta t_{1} \cdot L\left(\phi^{n}\right)$.

- Step 2. Compute $\phi^{n+1}=\phi^{n+1 / 2}+\Delta t_{2} \cdot \Delta \phi^{n+1 / 2}+\Delta t_{3} \cdot\left(\phi^{n+1 / 2}-\phi^{n}\right)$.

Remark 1. In this two steps, $\Delta t_{1}$ is the time step of step 1. $\Delta t_{2}$ and $\Delta t_{3}$ represent the two time steps of step 2 . In step 1 , we obtain $\phi^{n+1 / 2}$ and then utilize it in step 2 , where $\Delta \phi^{n+1 / 2}$ represents the Laplacian operator that acts on the level set function. The third term of step 2 is a new restraint term to avoid updating the level set function seriously.

The purpose of this operator splitting method is significant. Owing to the execution of step 1, the contour evolves toward the object boundaries. After that, as a smoothing way, step 2 is extremely important as well for it eliminates the costly re-initialization procedure and avoids updating the level set function severely. As indicated in $[27,38]$, the evolution of a function with its Laplacian is equivalent to a Gaussian filtering process to regularize the level set function. Thus, step 2 plays a natural role for smoothing the level set function and maintaining its stability. Actually, as a following procedure of step 1 , step 2 can be influenced by step 1 at the same time. If the level set function is too steep, it needs to properly increase $\Delta t_{2}$ so as to smooth more. On the other hand, step 2 has a direct impact on step 1 . It is just the mutual cooperation of these two steps that promotes the steady evolution of the level set function and reduces the computational complexity. More significantly, this operator splitting method can be easily extended to other related level set methods based on partial differential equations.

\subsection{An extension on vector-valued images}

As the vector-valued $\mathrm{C}-\mathrm{V}$ model [25], the extension of our model on vector-valued images is natural. Similarly to the analysis in Subsection 3.1, we calculate each channel singly and combine each other to control the contour evolution. Specifically, for $i=1, \cdots, N$, we define $c_{i}^{+L}$ and $c_{i}^{-L}$ as

$$
\begin{aligned}
& c_{i}^{+L}=\operatorname{mean}\left(I_{i} \in\left(\{(x, y) \in \Omega \mid \phi(x, y)>0\} \cap W_{k}(x, y)\right)\right), \\
& c_{i}^{-L}=\operatorname{mean}\left(I_{i} \in\left(\{(x, y) \in \Omega \mid \phi(x, y)<0\} \cap W_{k}(x, y)\right)\right),
\end{aligned}
$$

where $I_{i}$ is the $i$ th channel of the image.

Next, a natural idea is to consider using $c_{i}^{+L}$ and $c_{i}^{-L}$ to replace $c_{i}^{+}$and $c_{i}^{-}$, thus the level set formulation (10) of the vector-valued $\mathrm{C}-\mathrm{V}$ model can be further rewritten as

$$
\begin{aligned}
\frac{\partial \phi}{\partial t}= & \delta(\phi)\left[\mu \operatorname{div}\left(\frac{\nabla \phi}{|\nabla \phi|}\right)-\frac{1}{N} \sum_{i=1}^{N} \lambda_{i}^{+}\left(I_{i}(x, y)\right.\right. \\
& \left.\left.-c_{i}^{+L}\right)^{2}+\frac{1}{N} \sum_{i=1}^{N} \lambda_{i}^{-}\left(I_{i}(x, y)-c_{i}^{-L}\right)^{2}\right] .
\end{aligned}
$$

From the construction of Equation 17, the evolving contour is driven by the local region force. As a result of this replacement, all the local region information in every channel of the vector-valued image is integrated with each other, which is beneficial to detecting the object boundaries. In addition, it can also avoid some limitations of using a single channel for the vector-valued images.

\subsection{Numerical implementation}

In numerical implementation, as discussed in [39], the second time step $\Delta t_{2}$ should be set small, which can reduce the risk of moving the zero level set away from its original location. $\Delta t_{1}$ is related to updating of the level set function and has an impact on its smoothness. Generally, the choices of this two time steps should be comparable with $\Delta t_{2}<\Delta t_{1}$. Furthermore, except for maintaining the smoothness and numerical stability, choosing a small $\Delta t_{2}$ is reasonable for avoiding the emergence of boundary leakage problems. Similarly, $\Delta t_{3}$ should be selected small for maintaining the stability of the level set function satisfactorily.

For the convenience of calculation, as mentioned in [7,17], the Heaviside function $H$ and the Dirac function $\delta$ are usually approximated by a smooth function $H_{\varepsilon}$ and its derivative $\delta_{\varepsilon}$ as

$$
\begin{aligned}
H_{\varepsilon}(z) & =\frac{1}{2}\left[1+\frac{2}{\pi} \arctan \left(\frac{z}{\varepsilon}\right)\right], \\
\delta_{\varepsilon}(z) & =\frac{1}{\pi} \frac{\varepsilon}{\varepsilon^{2}+z^{2}} .
\end{aligned}
$$


Summarizing the descriptions on Subsections 3.1, 3.2, and 3.3 , the main steps of our method for image segmentation are presented as follows:

1. Input an original image $I$. Initialize the level set function $\phi$, where we can choose a signed distance function $[7,25]$ or a binary function $[27,35,36,39]$.

2. If $I$ is a gray scale image, compute $c_{1}^{\mathrm{L}}$ and $c_{2}^{\mathrm{L}}$ by Equations 11 and 12 . On the other hand, if $I$ is a vector-valued image, compute $c_{i}^{+L}$ and $c_{i}^{-L}$ by Equations 15 and 16.

3. Implement the presented operator splitting method in Subsection 3.2 sequentially.

4. Check whether the level set function satisfies the stationary condition. If not, return to step 2 .

Remark 2. In step 2, we first need to judge whether the imputing image is a gray scale image or not, where we can test it with the help of some simple experiments, such as the MATLAB program (MathWorks Inc, Natick, MA, USA). Especially, in most cases, the color image can be distinguished by direct observation.

Remark 3. Our method is different from the methods in $[41,42]$. Even if the authors also introduced the local region model by employing the maximum a posteriori estimation and Parzen method, they mainly focused on the statistical interpretation and application of the Mumford-Shah model. Besides, they approximated it from a maximum a posteriori model where each region is modeled by the mean estimated in a local Gaussian neighborhood. However, focusing on the improvement of the $\mathrm{C}-\mathrm{V}$ model, our method use the local region information to replace the global region information and present an operator splitting method for implementation. Furthermore, our method is easily extended to the vector-valued images.
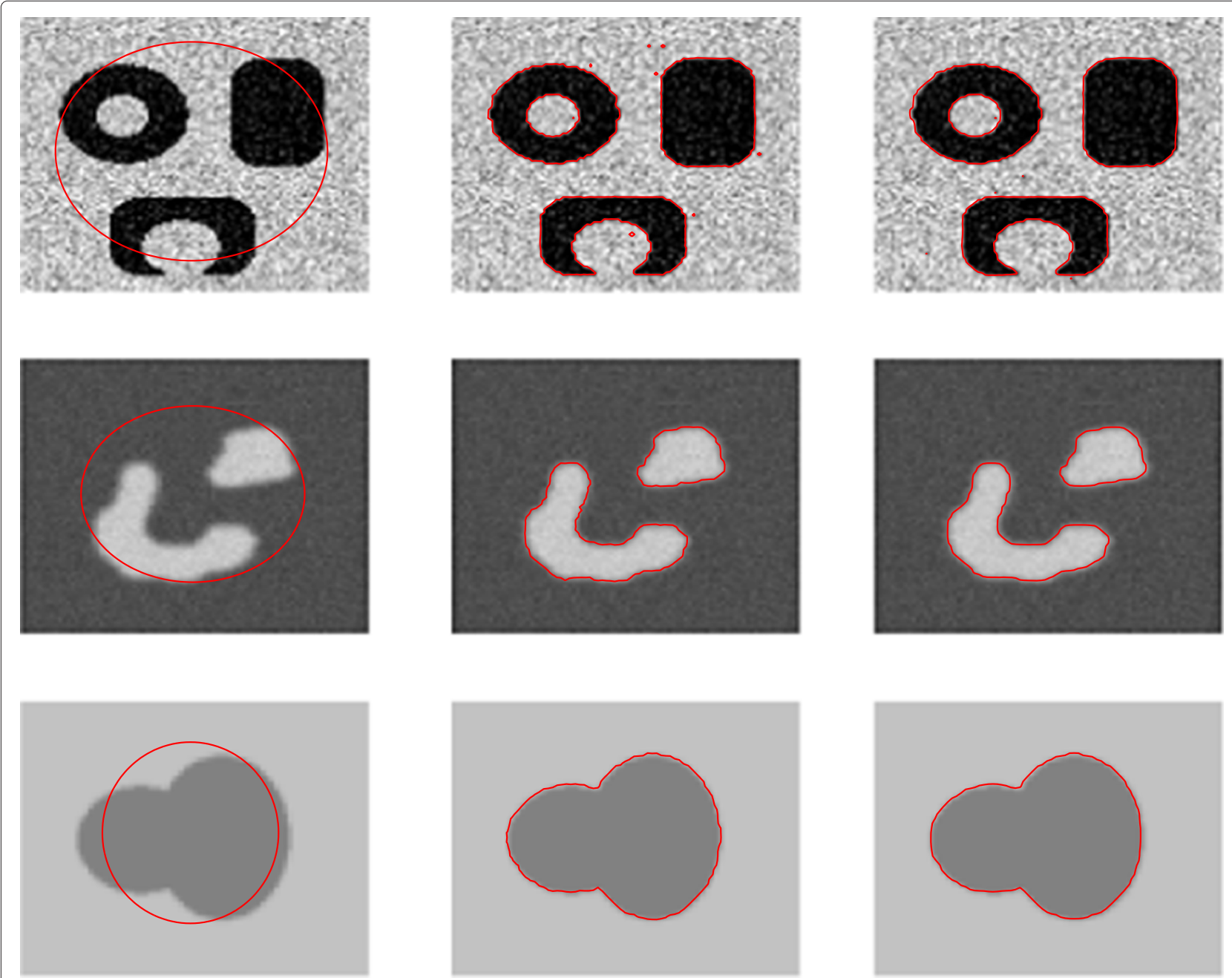

Figure 1 Comparison results for the synthetic images. Column 1, initial contours. Column 2, results of the C-V model. Column 3, results of our method. 

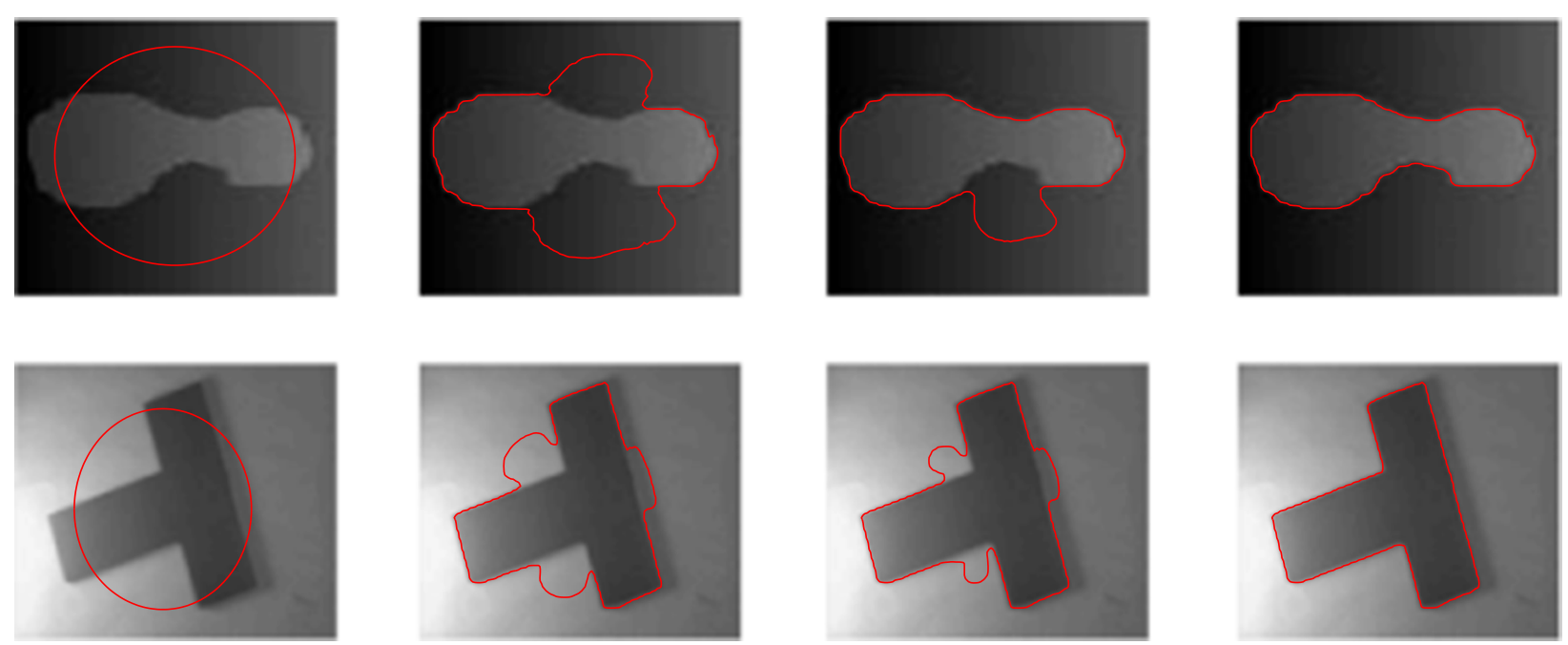

Figure 2 The evolving process and the final results of our method. The evolving process and the final results of our method with application to the images with intensity inhomogeneity. There are initial contour, middle results and final result from left to right in every row.

\section{Experimental results}

In this section, a series of synthetical and real-world images are used to test the effectiveness and performance of our method. All the experiments are implemented in Matlab 7.0 on a personal computer with Intel Pentium D (Intel Corp, Sta. Clara, CA, USA) CPU $3.00 \mathrm{GHz}$ and $1 \mathrm{~GB}$ of memory. We choose the size of the truncated Gaussian window as $4 k+1$ by $4 k+1$, where $k$ is the greatest integer smaller than the standard deviation $\sigma$. Unless otherwise specified, the default parameters are set as $\Delta t_{1}=0.1$, $\Delta t_{2}=0.01, \Delta t_{3}=0.01, \varepsilon=1.0$, and $\lambda_{1}=\lambda_{2}=1.0$, and for $i=1, \cdots, N, \lambda_{i}^{+}=\lambda_{i}^{-}=1.0$. Besides, the parameters $\sigma$ and $\mu$ should be set as different values according to the image characteristics, such as intensity, shape, and color.

Comparing with the $\mathrm{C}-\mathrm{V}$ model in the beginning, we test the performance of our method on some synthetic images. Figure 1 shows the comparison results of the $\mathrm{C}-\mathrm{V}$ model and our method. In row 1 and row 2, the two test images are with noise. With the same initial contours, the comparison results illustrate that our method can obtain similar performances as the $\mathrm{C}-\mathrm{V}$ model. Besides, our method performs well for the noisy image with lowintensity inhomogeneity. In our method, we choose $\sigma=$ 10.0, $\mu=0.1 \times 255^{2}$ in the first image, and $\sigma=3.0$, $\mu=0.005 \times 255^{2}$ in the last two images.
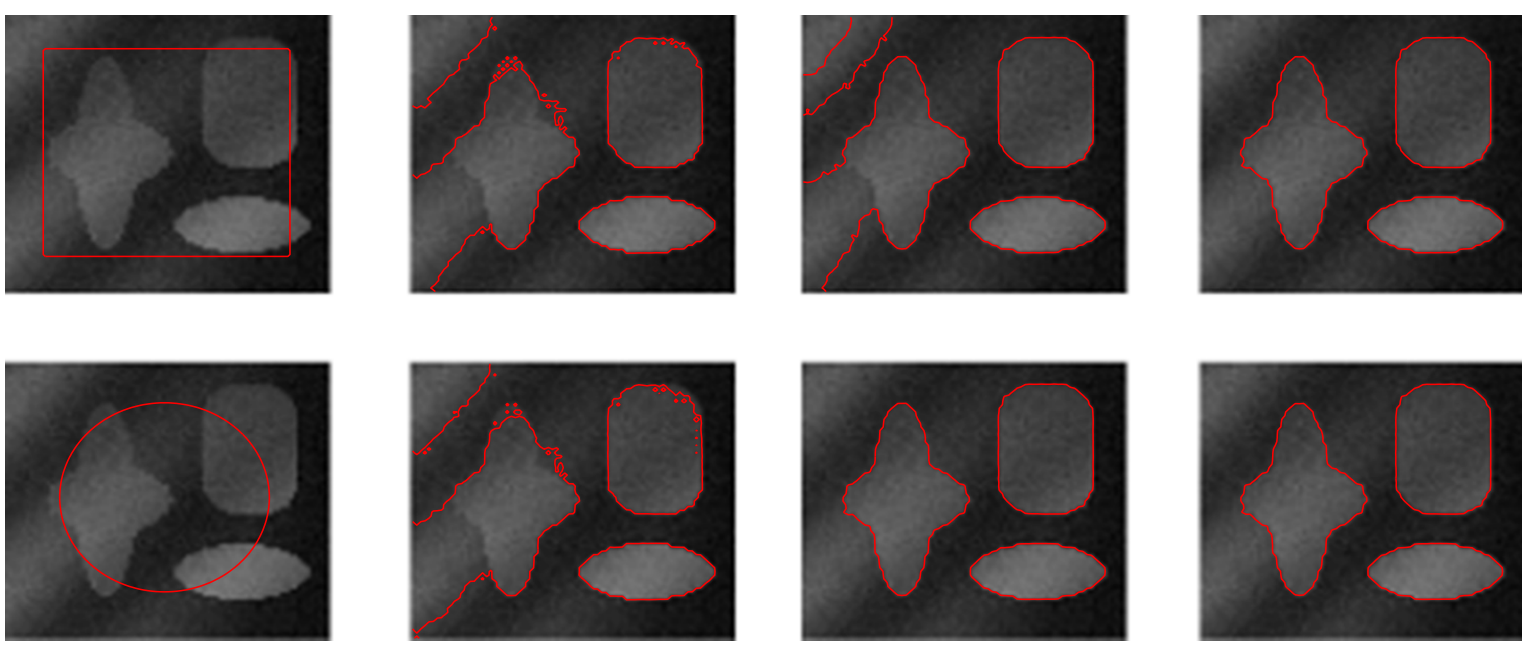

Figure 3 Comparison results for a synthetical image with different initial contours. Column 1, initial contours. Column 2, results of the C-V model. Column 3, results of the LBF model $[16,17]$. Column 4, results of our method. 
Table 1 Iterations and CPU time (in seconds) of two different kinds of level set initialization in Figure 3

\begin{tabular}{lcccccc}
\hline & \multicolumn{2}{c}{ Row $\mathbf{1}$} & & \multicolumn{2}{c}{ Row $\mathbf{2}$} \\
\cline { 2 - 3 } \cline { 6 - 6 } & Iteration & Time (s) & & Iteration & Time (s) \\
\hline C-V & 300 & 8.05 & & 300 & 8.80 \\
LBF $[16,17]$ & 200 & 8.39 & & 850 & 32.53 \\
Our method & 180 & 9.08 & & 1500 & 71.49 \\
\hline
\end{tabular}

Figure 2 shows our method with application to the images with intensity inhomogeneity. In every row, there are the initial contour, middle results, and the final result from left to right. The evolving process indicates that the contour is driven by the local region fitting energy and moves toward the object boundaries. The results illustrate that our method has a satisfying performance to deal with the problem of intensity inhomogeneity, which demonstrates the advantage of the introduction of the local image region information. Here, we set $\sigma=10.0, \mu=0.001 \times$ $255^{2}$ in the first image, and $\sigma=3.0, \mu=0.003 \times 255^{2}$ in the second image.

In Figure 3, we show the comparison results of the $\mathrm{C}-\mathrm{V}$ model, the LBF model, and our method. The test image is a typical synthetic image with intensity inhomogeneity [17]. We choose two different initial contours, that is, a square curve in the first row and a cycle curve in the second row. All the parameters in the $\mathrm{C}-\mathrm{V}$ model and the LBF model are tried many times and selected for the best performance. In the first row, the level set function initialization is a binary function. By relying on the global region information, the $\mathrm{C}-\mathrm{V}$ model cannot segment the image with intensity inhomogeneity satisfactorily. The LBF model is sensitive to the location of the initial contour [30], and therefore it fails in detecting the object boundaries. It is clear that only our method obtains desirable results. In the second row, even if we initialize the level set function as a signed distance function, the $\mathrm{C}-\mathrm{V}$ model still performs badly. However, the LBF model and our method detect the object boundaries successfully. In our method, we set $\sigma=5.0, \mu=$ $0.003 \times 255^{2}$. All the iterations and CPU time are presented in Table 1. From the comparison results in Figure 3 and Table 1 , we can easily observe that the different kinds of the level set initialization for our method still lead to similar segmentation results, which is better than the LBF model. In addition, in our method, different initial contours need different iterations and time. As a whole, our method spends more time than the $\mathrm{C}-\mathrm{V}$ model and the LBF model.

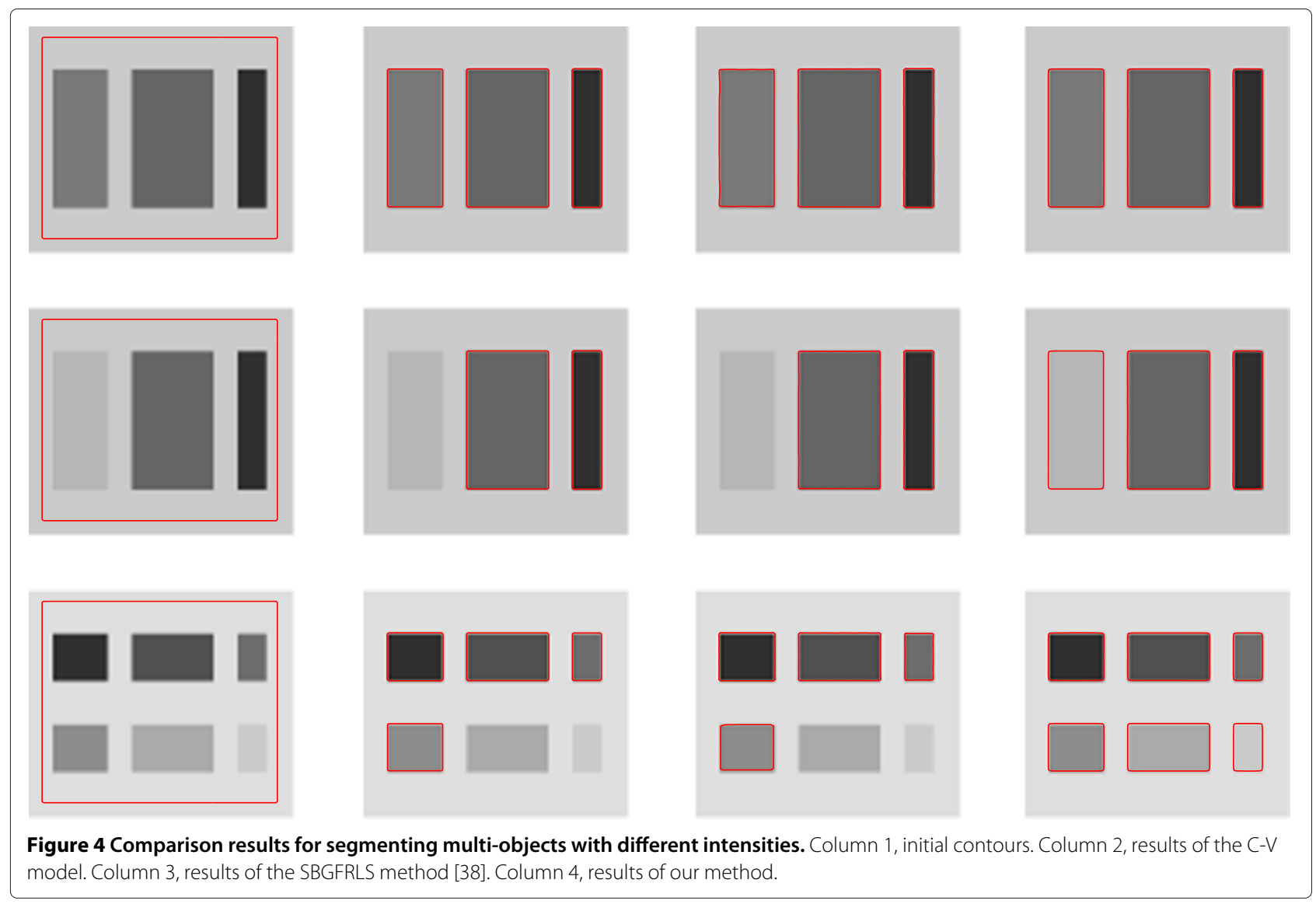


Table 2 Iterations and CPU time (in seconds) for segmenting multi-objects with different intensities in Figure 4

\begin{tabular}{|c|c|c|c|c|c|c|}
\hline & \multicolumn{2}{|c|}{ Row 1} & \multicolumn{2}{|c|}{ Row 2} & \multicolumn{2}{|c|}{ Row 3} \\
\hline & Iteration & Time (s) & Iteration & Time (s) & Iteration & Time (s) \\
\hline$C-V$ & 120 & 3.50 & 120 & 3.25 & 150 & 4.08 \\
\hline SBGFRLS [38] & 80 & 1.91 & 80 & 1.94 & 100 & 2.35 \\
\hline Our method & 140 & 11.58 & 140 & 11.24 & 180 & 15.65 \\
\hline
\end{tabular}

Figure 4 shows that the comparison results for some synthetic images, which have multi-objects with different intensities. We compare our method with the $\mathrm{C}-\mathrm{V}$ model and the selective binary and Gaussian filtering regularized level set (SBGFRLS) method [38]. In the first row, as the same experiment to be tested in [38], the background intensity is 200 , and the three objects are 120,100 , and 50 from left to right, where all three methods extract objects successfully. In the second row, the left object intensity is 180 , which is close to the background intensity. As discussed in [38], the results of the C-V model and the SBGFRLS method are unsatisfactory because the left object with intensity 180 is not extracted. The most important reason is that the two methods mainly depend on the global region information. However, because of introducing the local image region information effectively, our method extracts all the objects successfully. Moreover, in the third row, the background intensity is 220, and the intensities of six objects are 50, 80, 110, 140, 170, and 200 from left to right and top to bottom, respectively. The C-V model and the SBGFRLS method fail in extracting two objects with intensities 170 and 200 in the bottom. However, our method extracts all the objects as before. Therefore, owing to the local property, our method performs well for segmenting the multi-objects with different intensities. All the iterations and CPU time are shown in Table 2, from which we can see that our method takes more iterations and CPU time than other two methods. Even so, our method is very competitive for it can satisfactorily segment multi-objects with different intensities. We choose $\sigma=6.0, \mu=0.001 \times 255^{2}$ in our method.

The next experiments are focused on demonstrating the effectiveness of our method with application to the vector-valued images. As a special example, we use the color image. By dividing the image into three channels using red, green, and blue colors, one can extract objects that normally undetectable when the color image is transformed to a scalar intensity image [25]. Figure 5 takes on the evolving process of the contours and the final results of two real-world color images, which specifies that our method can extract the objects clearly. We set $\sigma=3.0$, $\mu=0.01 \times 255^{2}$.

In comparing with the vector-valued C-V model [25], Figure 6 shows three examples to illustrate the performance between our method and the vector-valued $\mathrm{C}-\mathrm{V}$ model with application to the color images. From the comparison results, it is clear that the performance of the two kinds of methods are roughly similar, but there are some difference in details. Because of the global property, the vector-valued $\mathrm{C}-\mathrm{V}$ model extracts all the prominent objects of the images. However, for the real-world images
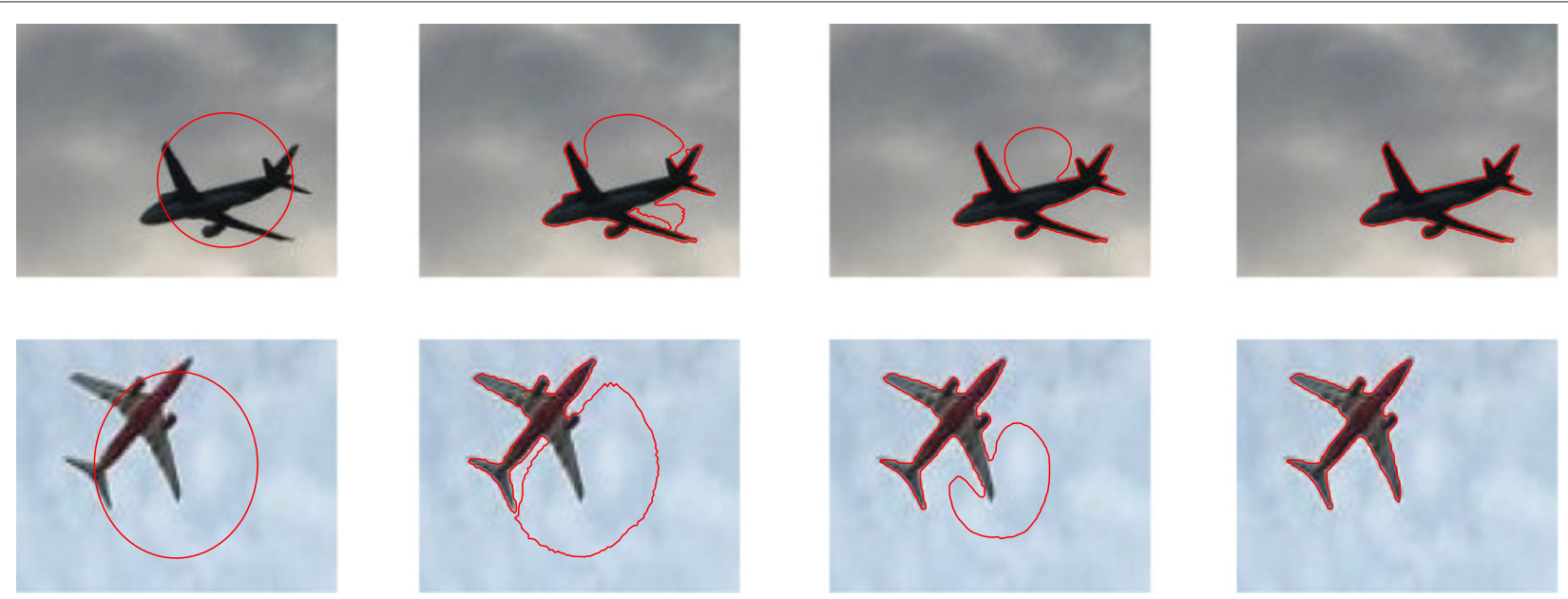

Figure 5 Evolving process and the final results of our method with application to real-world color images. There are initial contour and middle and final results from left to right in every row. 


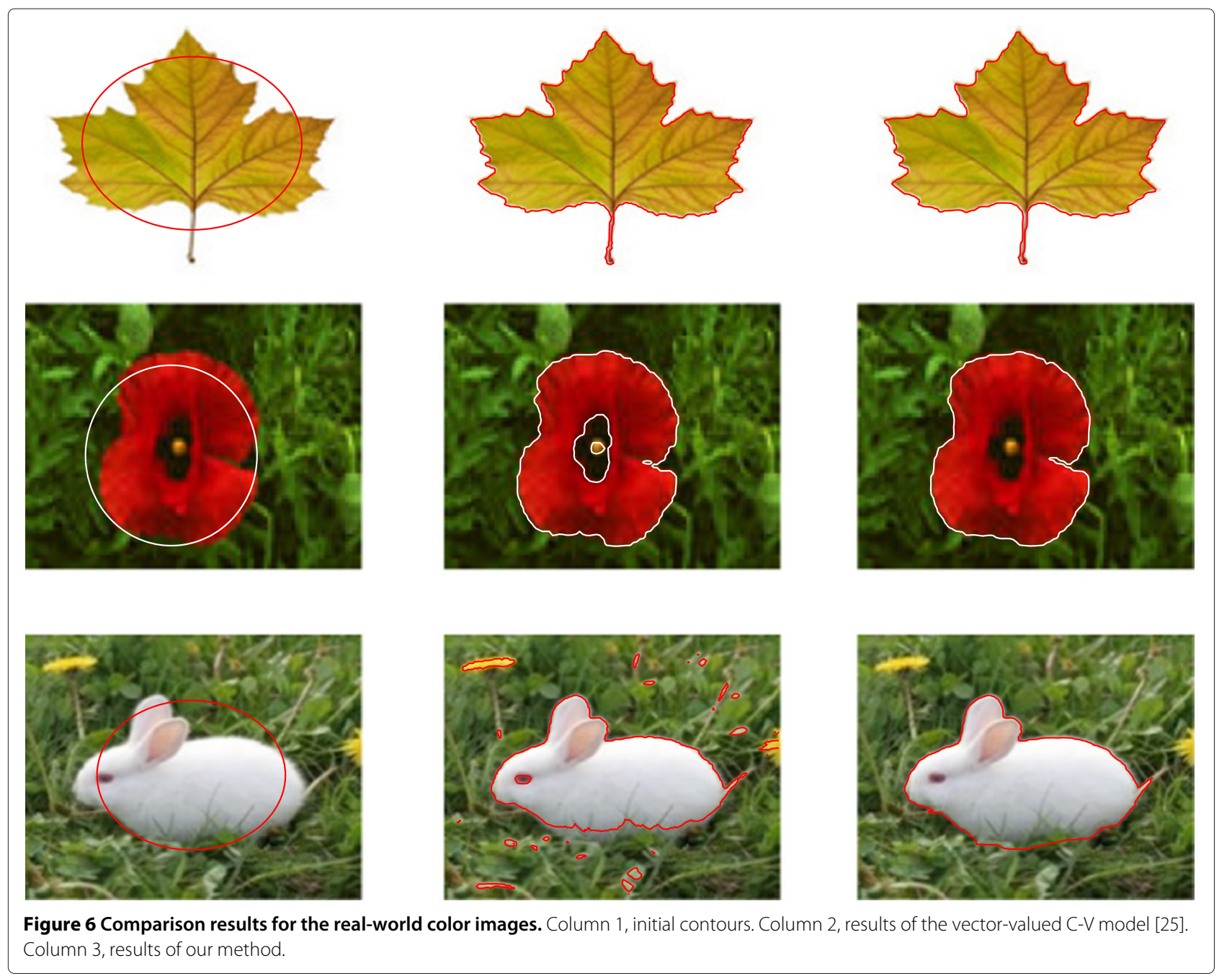

with complicated background, we usually pay attention to some big or desirable objects. Thus, we can consider to initialize the contours near the object boundaries; and largely owing to the influence of the local image region information, our method obtains more desirable results. To be specific, for the leaf image with single object in the first row, the two kinds of results are similar to each other.
The second image with complex background, comparing with the vector-valued $\mathrm{C}-\mathrm{V}$ model, our method demonstrates better in visual perception. In the third image, a major concern is the rabbit in the grass. Therefore, the comparison results illustrate that our method performs better than the vector-valued $\mathrm{C}-\mathrm{V}$ model. We choose $\sigma=$ 3.0 in our method and with the same other parameters

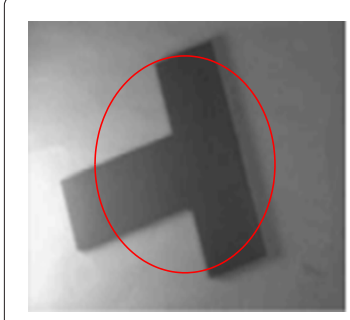

(a)

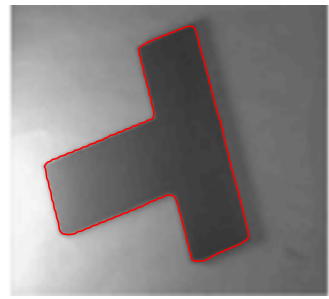

(b)

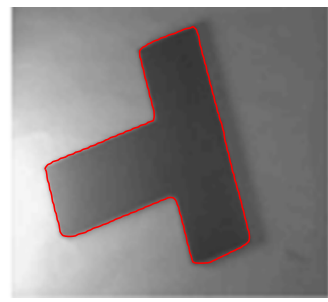

(c)

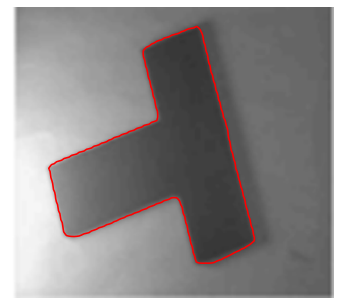

(d)

Figure 7 Comparison results for different values of the standard deviation $\sigma$. (a) Initial contours. (b) The result of $\sigma=2$.0. (c) The result of $\sigma=5.0$. (d) The result of $\sigma=8.0$. 
as the vector-valued $\mathrm{C}-\mathrm{V}$ model. Specifically, we set $\mu=$ $0.01 \times 255^{2}$ in the first row and the second row, and $\mu=0.05 \times 255^{2}$ in the last row.

To further test the sensitivity of the proposed method to the standard deviation of the truncated Gaussian window, we design several different values for $\sigma$, and their results are presented in Figure 7. We borrow the same image as in Figure 2, where $\sigma=3.0$. Though we choose different values, the results are similar and satisfying, which demonstrates that our method is less sensitive to the choice of $\sigma$. We set $\mu=0.01 \times 255^{2}$.

\section{Conclusion}

In this paper, we have proposed an efficient operator splitting method for local region $\mathrm{C}-\mathrm{V}$ model. By introducing the window function, we increased the local image region information to improve the $\mathrm{C}-\mathrm{V}$ model, which performs better than the traditional $\mathrm{C}-\mathrm{V}$ model on segmenting images with intensity inhomogeneity. In order to regularize the level set function and maintain the numerical stability during the level set evolution, we presented an operator splitting method. In this method, we employed the Laplacian operator to act on the level set function and increased a new restraint term to prevent updating the level set function seriously. Comparing with other related methods $[7,17,42]$, the motivation and superiority of our method have been discussed in details. Furthermore, our method has been extended to the vector-valued image segmentation, such as the color image. Our method can effectively eliminate the re-initialization procedure and ensure the numerical calculation stability. A large number of numerical experiments have been used to test and demonstrate that our method can effectively segment the gray scale images with intensity inhomogeneity and multiobjects with different intensities, and perform well on the real-world color images.

\section{Competing interests}

The authors declare that they have no competing interests.

\section{Author details \\ 1 School of Mathematical Sciences, University of Electronic Science and Technology of China, Chengdu, Sichuan 611731, China. ${ }^{2}$ Department of Mathematics and Computer Science, Anshun University, Anshun, Guizhou 561000, China.}

\section{Acknowledgements}

The authors would like to thank the editor and the anonymous referee for their valuable comments and suggestions, which are very helpful for revising and improving this paper. This research is supported by NSFC (no. 61170311), Chinese Universities Specialized Research Fund for the Doctoral Program (20110185110020), Sichuan Provincial Department of Science \& Technology Research Project (no. 12ZC1802), and the Fundamental Research Funds for the Central Universities (no. 09CX04003A).

\section{References}

1. D Cremers, M Rousson, R Deriche, A review of statistical approaches to level set segmentation: integrating color, texture, motion and shape. Int. J. Comput. Vis. 72(2), 195-215 (2007)

2. A Mitiche, IB Ayed, Variational and level set methods in image segmentation. (Springer-Verlag, Berlin,Heidelberg, 2010)

3. M Kass, A Witkin, D Terzopoulos, Snakes: active contour models. Int. J. Comput. Vis. 1(4), 321-331 (1988)

4. SOsher, JA Sethian, Fronts propagating with curvature-dependent speed: algorithms based on Hamilton-Jacobi formulation. J. Comput. Phys. 79(1), 12-49 (1988)

5. V Caselles, F Catte, T Coll, F Dibos, A geometric model for active contours in image processing. Numer. math. 66(1), 1-31 (1993)

6. V Caselles, R Kimmel, G Sapiro, Geodesic active contours. Int. J. Comput. Vis. 22(1), 61-79 (1997)

7. TF Chan, LA Vese, Active contours without edges. IEEE Trans. Image Process. 10(2), 266-277 (2001)

8. J Park, J Keller, Snakes on the watershed. IEEE Trans. Pattern Anal. Mach. Intell. 23(10), 1201-1205 (2001)

9. R Goldenberg, R Kimmel, E Rivlin, M Rudzsky, Fast geodesic active contours. IEEE Trans. Image Process. 10(10), 1467-1475 (2001)

10. A Vasilevskiy, K Siddiqi, Flux-maximizing geometric flows. IEEE Trans. Pattern Anal. Mach. Intell. 24(12), 1565-1578 (2002)

11. $Y$ Xiang, A Chung, J Ye, An active contour model for image segmentation based on elastic interaction. J. Comput. Phys. 219(1), 455-476 (2006)

12. C Samson, L Blanc-Feraud, G Aubert, J Zerubia, A level set model for image classification. Int. J. Comput. Vis. 40(3), 187-197 (2000)

13. A Tsai, A Yezzi, AS Willsky, Curve evolution implementation of the Mumford-Shah functional for image segmentation, denoising, interpolation, and magnification. IEEE Trans. Image Process. 10(8), 1169-1186 (2001)

14. J Kim, J Fisher, A Yezzi, M Cetin, A Willsky, A nonparametric statistical method for image segmentation using information theory and curve evolution. IEEE Trans. Image Process. 14(10), 1486-1502 (2005)

15. C A Sarti, E Corsi, C Mazzini, Lamberti, Maximum likelihood segmentation of ultrasound images with Rayleigh distribution. IEEE Trans. Ultrason. Ferroelectr. Freq. Control. 52(6), 947-960 (2005)

16. C Li, JC Kao, Z Gore, Ding, Implicit active contours driven by local binary fitting energy, in Proceedings of the IEEE Conference on Computer Vision and Pattern Recognition (IEEE Press. USA, 1-7 (2007)

17. C Li, C Kao, JC Gore, Z Ding, Minimization of region-scalable fitting energy for image segmentation. IEEE Trans. Image Process. 17(10), 1940-1949 (2008)

18. D Mumford, J Shah, Optimal approximations by piecewise smooth functions and associated variational problems. Commun. Pure Appl. Math. 42(5), 577-685 (1989)

19. J Lie, M Lysaker, X Tai, A variant of the level set method and applications to image segmentation. Math. Comp. 75(255), 1155-1174 (2006)

20. J Lie, M Lysaker, X Tai, A binary level set model and some applications to Mumford-Shah image segmentation. IEEE Trans. Image Process. 15(5), 1171-1181 (2006)

21. TF Chan, S Esedoglu, M Nikolova, Algorithms for finding global minimizers of image segmentation and denoising models. SIAM. J. Appl. Math. 66(5), 1632-1648 (2006)

22. X Bresson, S Esedoglu, P Vandergheynst, J Thiran, S Osher, Fast global minimization of the active contour/snake model. J. Math. Imaging Vis. 28(2), 151-167 (2007)

23. E Bae, J Yuan, X Tai, Global minimization for continuous multiphase partitioning problems using a dual approach. Int. J. Comput. Vis. 92(1), 112-129 (2009)

24. LA Vese, TF Chan, A multiphase level set framework for image segmentation using the Mumford and Shah model. Int. J. Comput. Vis. 50(3), 271-293 (2002)

25. TF Chan, BY Sandberg, LA Vese, Active contours without edges for vector-valued images. J. Visual Communic. Imag. Representation. 11(2), 130-141 (2000)

26. L Wang, L He, A Mishra, C Li, Active contours driven by local Gaussian distribution fitting energy. Signal Process. 89(12), 2435-2447 (2009) 
27. K Zhang, $\mathrm{H}$ Song, L Zhang, Active contours driven by local image fitting energy. Pattern Recognit. 43(4), 1199-1206 (2010)

28. S Lankton, A Tannenbaum, Localizing region-based active contours. IEEE Trans. Image Process. 17(11), 2029-2039 (2008)

29. WTao, XTai, Multiple piecewise constant with geodesic active contours (MPC-GAC) framework for interactive images segmentation using graph cut optimization. Image Vis. Comput. 29(8), 499-508 (2011)

30. X Wang, D Huang, $\mathrm{HXu}$, An efficient local Chan-Vese model for image segmentation. Pattern Recognit. 43(3), 603-618 (2010)

31. M Sussman, P Smereka, S Osher, A level set approach for computing solutions to incompressible two-phase flow. J. Comput. Phys. 119(1), 146-159 (1994)

32. M Sussman, E Fatemi, An efficient, interface preserving level set redistancing algorithm and its application to interfacial incompressible fluid flow. SIAM J. Sci. Comput. 20(4), 1165-1191 (1999)

33. J Gomes, O Faugeras, Reconciling distance functions and level sets. J. Visual Communic. Imag. Representation. 11(2), 209-223 (2000)

34. D Peng, B Merriman, S Osher, H Zhao, M Kang, A PDE-based fast local level set method. J. Comput. Phys. 155(2), 410-438 (1999)

35. C Li, CXu, C Gui, MD Fox, in Proceedings of the IEEE Conference on Computer Vision and Pattern Recognition. Level set evolution without re-initialization: a new variational formulation (IEEE Press, USA, 2005), pp. 430-436

36. C Li, CXu, C Gui, MD Fox, Distance regularized level set evolution and its application to image segmentation. IEEE Trans. Image Process. 19(12), 3243-3254 (2010)

37. XXie, Active contouring based on gradient vector interaction and constrained level set diffusion. IEEE Trans. Image Process. 19(1), 154-164 (2010)

38. K Zhang, L Zhang, H Song, W Zhou, Active contours with selective local or global segmentation: a new formulation and level set method. Image Vis. Comput. 28(4), 668-676 (2010)

39. K Zhang, L Zhang, H Song, D Zhang, Re-initialization free level set evolution via reaction diffusion. IEEE Trans. Image Process. 22(1), 258-271 (2013)

40. J Shen, A stochastic-variational model for soft Mumford-Shah segmentation. Int. J. Biomed. Imaging. 2006, 1-14 (2006)

41. T Brox, D Cremers, in Proceedings of the 1st International Conference on Scale Space and Variational Methods in Computer Vision. On the statistical interpretation of the piecewise smooth Mumford-Shah functional (Springer, Berlin, 2007), pp. 203-213

42. T Brox, D Cremers, On local region models and a statistical interpretation of the piecewise smooth Mumford-Shah functional. Int. J. Comput. Vis. 84(2), 184-193 (2009)

doi:10.1186/1687-6180-2013-97

Cite this article as: Wang et al: An efficient operator splitting method for local region Chan-Vese model. EURASIP Journal on Advances in Signal Processing 2013 2013:97.

\section{Submit your manuscript to a SpringerOpen ${ }^{\mathcal{O}}$ journal and benefit from:}

- Convenient online submission

- Rigorous peer review

- Immediate publication on acceptance

- Open access: articles freely available online

- High visibility within the field

- Retaining the copyright to your article

Submit your next manuscript at $\boldsymbol{\wedge}$ springeropen.com 